\title{
PARTIAL DYNAMICAL SYMMETRY IN A FERMIONIC MANY-BODY SYSTEM
}

\author{
JUTTA ESCHER \\ TRIUMF, 4004 Wesbrook Mall, Vancouver, B.C. V6T 2A3, Canada \\ E-mail: escher@triumf.ca
}

\begin{abstract}
The concept of partial symmetry is introduced for an interacting fermion system. The associated Hamiltonians are shown to be closely related to a realistic nuclear quadrupole-quadrupole interaction. An application to ${ }^{12} \mathrm{C}$ is presented.
\end{abstract}

\section{Introduction}

The fundamental concept underlying algebraic theories in quantum physics is that of an exact or dynamical symmetry. Realistic quantum systems, however, often require the associated symmetry to be broken in order to allow for a proper description of some observed basic features. Partial dynamical symmetry (PDS) describes an intermediate situation in which some eigenstates exhibit a symmetry which the associated Hamiltonian does not share. The objective of this approach is to remove undesired constraints from the theory while preserving the useful aspects of a dynamical symmetry, such as solvability, for a subset of eigenstates. 1 . Here we present an example of a PDS in an interacting fermion system. In the symplectic shell model of nuclei, 3 we introduce PDS Hamiltonians which are closely related to the nuclear quadrupole-quadrupole interaction. An application to ${ }^{12} \mathrm{C}$ is discussed.

\section{PDS Hamiltonians and quadrupole-quadrupole interaction}

The quadrupole-quadrupole interaction is an important ingredient in models that aim at reproducing quadrupole collective properties of nuclei. A model which is able to fully accommodate the action of the collective quadrupole operator, $Q_{2 m}=\sqrt{16 \pi / 5} \sum_{s} r_{s}^{2} Y_{2 m}\left(\hat{r}_{s}\right)$, is the symplectic shell model (SSM), an algebraic scheme which respects the Pauli principle 3 In the SSM, this operator takes the form $Q_{2 m}=\sqrt{3}\left(\hat{C}_{2 m}^{(11)}+\hat{A}_{2 m}^{(20)}+\hat{B}_{2 m}^{(02)}\right)$, where $\hat{A}_{l m}^{(20)}, \hat{B}_{l m}^{(02)}$, and $\hat{C}_{l m}^{(11)}$ are symplectic generators with good $\mathrm{SU}(3)$ [superscript $(\lambda, \mu)$ ] and $\mathrm{SO}(3)$ [subscript $l, m]$ tensorial properties. The $\hat{A}_{l m}^{(20)}\left(\hat{B}_{l m}^{(02)}\right), l=0$ or 2 , create (annihilate) $2 \hbar \omega$ excitations in the system. The $\hat{C}_{l m}^{(11)}, l=1$ or 2 , generate a $\mathrm{SU}(3)$ subgroup and act only within one harmonic oscillator (h.o.) shell $\left(\sqrt{3} \hat{C}_{2 m}^{(11)}=Q_{2 m}^{E}\right.$, the quadrupole operator of Elliott, which does not couple 
different h.o. shells, 1 and $\hat{C}_{1 m}^{(11)}=\hat{L}_{m}$, the angular momentum operator). The symplectic basis is generated by repeated application of $\hat{A}^{(20)}$ to a $0 \hbar \omega$ shell model configuration, labeled by its Elliott quantum numbers $\left(\lambda_{\sigma}, \mu_{\sigma}\right)$. The resulting $N \hbar \omega$ excited states $(N=0,2, \ldots)$ are coupled to good $\mathrm{SU}(3) \supset \mathrm{SO}(3)$ symmetry $(\lambda, \mu) \kappa L M$, where $\kappa$ enumerates multiple occurrences of $L$ in the $\mathrm{SU}(3)$ irrep $(\lambda, \mu)$. This labeling scheme defines a dynamical symmetry basis.

The quadrupole-quadrupole interaction connects h.o. states differing in energy by $0 \hbar \omega, \pm 2 \hbar \omega$, and $\pm 4 \hbar \omega$, and may be written as

$$
\begin{aligned}
Q_{2} \cdot Q_{2}= & 9 \hat{C}_{S U 3}-3 \hat{C}_{S p 6}+\hat{H}_{0}^{2}-2 \hat{H}_{0}-3 \hat{L}^{2}-6 \hat{A}_{0} \hat{B}_{0} \\
& +\{\text { terms coupling different h.o. shells }\}
\end{aligned}
$$

where $\hat{C}_{S U 3}$ and $\hat{C}_{S p 6}$ are Casimir invariants of $\mathrm{SU}(3)$ and $\mathrm{Sp}(6, \mathrm{R})$. These operators, as well as $\hat{H}_{0}$ and $\hat{L}^{2}$, are diagonal in the dynamical symmetry basis. Unlike the Elliott quadrupole-quadrupole interaction, $Q_{2} \cdot Q_{2}$ breaks $\mathrm{SU}(3)$ symmetry within each h.o. shell since $\hat{A}_{0} \hat{B}_{0}$ mixes different $\mathrm{SU}(3)$ irreps. To study the action of $Q_{2} \cdot Q_{2}$ within such a shell, we consider the Hamiltonians

$$
\begin{aligned}
& H\left(\beta_{0}, \beta_{2}\right)=\beta_{0} \hat{A}_{0} \hat{B}_{0}+\beta_{2} \hat{A}_{2} \cdot \hat{B}_{2} \\
& =\frac{\beta_{2}}{18}\left(9 \hat{C}_{S U 3}-9 \hat{C}_{S p 6}+3 \hat{H}_{0}^{2}-36 \hat{H}_{0}\right)+\left(\beta_{0}-\beta_{2}\right) \hat{A}_{0} \hat{B}_{0} .
\end{aligned}
$$

For $\beta_{0}=\beta_{2}$, one recovers the dynamical symmetry, and for $\beta_{0}=12, \beta_{2}=18$, one obtains $Q_{2} \cdot Q_{2}=H\left(\beta_{0}=12, \beta_{2}=18\right)+\operatorname{const}(N)-3 \hat{L}^{2}+$ terms coupling different shells, where const $(N)$ is constant for a given h.o. $N \hbar \omega$ excitation.

For general $\beta_{0} \neq \beta_{2}, H\left(\beta_{0}, \beta_{2}\right)$ exhibits partial $\mathrm{SU}(3)$ symmetry: The Hamiltonian is not $\mathrm{SU}(3)$ invariant, yet it possesses a subset of 'special' states which respect the symmetry: All $0 \hbar \omega$ states are unmixed and span the entire $\left(\lambda_{\sigma}, \mu_{\sigma}\right)$ irrep. Moreover, among the excited configurations $(N>0)$, one finds additional states with good SU(3) symmetry. Unlike the $0 \hbar \omega$ states, however, they span only part of the corresponding SU(3) irreps. There are other states at each excited level which do not preserve the symmetry and therefore contain a mixture of irreps. The partial $\mathrm{SU}(3)$ symmetry of $H\left(\beta_{0}, \beta_{2}\right)$ is converted into partial dynamical symmetry by adding to it $\mathrm{SO}(3)$ rotation terms which lead to $L(L+1)$-type splitting but do not affect the wave functions. The solvable states then form rotational bands and since their wave functions are known one can evaluate their energies and the $\mathrm{E} 2$ rates between them analytically.

\section{Application to ${ }^{12} \mathrm{C}$}

To illustrate that the PDS Hamiltonians introduced here are physically relevant, we present an application to ${ }^{12} \mathrm{C}$. In Fig. 1, we compare the energy 


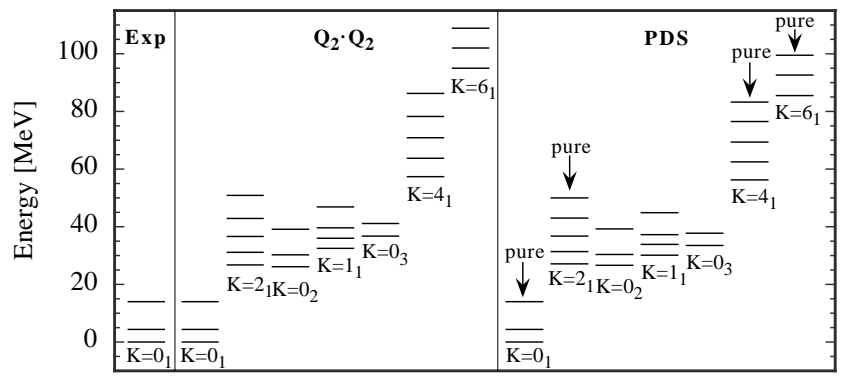

Figure 1. Energy spectra for ${ }^{12} \mathrm{C} . \mathrm{K}=0_{1}$ indicates the ground band in all three parts of the figure. In addition, resonance bands dominated by $2 \hbar \omega\left(\mathrm{K}=2_{1}, 0_{2}, 1_{1}, 0_{3}\right), 4 \hbar \omega\left(\mathrm{K}=4_{1}\right)$, and $6 \hbar \omega$ excitations $(K=61)$ are shown for the two calculations. The angular momenta of the states in the rotational bands are $L=0,2,4, \ldots$ for $\mathrm{K}=0$ and $L=\mathrm{K}, \mathrm{K}+1, \mathrm{~K}+2, \ldots$ otherwise.

spectra of $H_{P D S}=h(N)+\xi H\left(\beta_{0}=12, \beta_{2}=18\right)+\gamma_{2} \hat{L}^{2}+\gamma_{4} \hat{L}^{4}$ and $H_{Q \cdot Q}=\hat{H}_{0}-\chi Q_{2} \cdot Q_{2}+d_{2} \hat{L}^{2}+d_{4} \hat{L}^{4}$, where $h(N)$ is constant for a given $N \hbar \omega$ excitation. $H_{P D S}$ has families of pure $\mathrm{SU}(3)$ eigenstates which can be organized into rotational bands; they are indicated in the figure.

Although the PDS Hamiltonian cannot account for intershell correlations, it is able to reproduce various features of the quadrupole-quadrupole interaction, as can be seen in Fig. 2, where the structure of selected PDS eigenstates is compared to that of the corresponding $Q_{2} \cdot Q_{2}$ eigenstates: PDS eigenfunctions do not contain admixtures from different $N \hbar \omega$ configurations, but belong entirely to one level of excitation. We find that, for reasonable interaction parameters, the $N \hbar \omega$ level to which a particular PDS band belongs is also dominant in the corresponding band of exact $Q_{2} \cdot Q_{2}$ eigenstates. Moreover, within this dominant excitation, eigenstates of both Hamiltonians have similar SU(3) distributions. Structural differences, nevertheless, do arise and are reflected in the very sensitive interband transition rates.5 Overall, however, we may conclude that PDS eigenstates approximately reproduce the structure of the exact $Q_{2} \cdot Q_{2}$ eigenstates, for both ground and the resonance bands.

\section{Summary}

The notion of partial dynamical symmetries extends the familiar concepts of exact and dynamical symmetries. It is applicable when a subset of states exhibit a symmetry which does not arise from invariance properties of the relevant Hamiltonian. Recent studies, including the one presented here, show that partial symmetries are indeed realized in various quantum systems. 

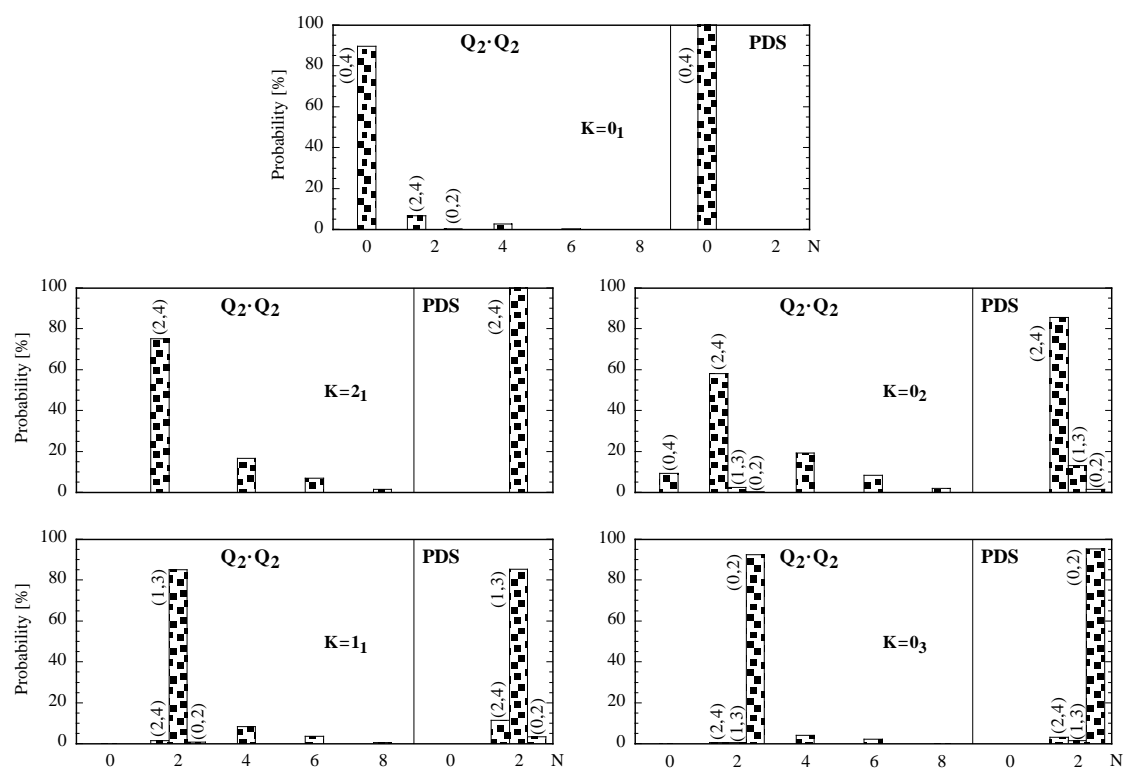

Figure 2. Decompositions for calculated $L^{\pi}=2^{+}$states of ${ }^{12} \mathrm{C}$. Individual contributions from the relevant $\mathrm{SU}(3)$ irreps at the $0 \hbar \omega$ and $2 \hbar \omega$ levels are shown for both the $8 \hbar \omega Q_{2} \cdot Q_{2}$ calculation and the PDS calculation. In addition, the total strengths contributed by the $N \hbar \omega$ excitations for $N>2$ are given for the $Q_{2} \cdot Q_{2}$ case.

\section{Acknowledgments}

This research was carried out in collaboration with A. Leviatan (Hebrew U.).

\section{References}

1. A. Leviatan, Phys. Rev. Lett. 77, 818 (1996); A. Leviatan and I. Sinai, Phys. Rev. C 60, 61301 (1999); A. Leviatan and J.N. Ginocchio, Phys. Rev. C 61, 24305 (2000).

2. J. Escher and A. Leviatan, Phys. Rev. Lett. 84, 1866 (2000).

3. G. Rosensteel and D.J. Rowe, Phys. Rev. Lett. 38, 10 (1977); Ann. Phys. 126, 343 (1980); D.J. Rowe, Rep. Prog. Phys. 48, 1419 (1985).

4. J.P. Elliott, Proc. Roy. Soc. A 245, 128 (1958); 245, 562 (1958).

5. J. Escher and A. Leviatan, to be submitted. 